\title{
Entrenamiento microquirúrgico básico para realizar un modelo animal de alotrasplante compuesto vascularizado*
}

\author{
Drs. ALEJANDRO RAMÍREZ M. ${ }^{1}$, RODRIGO CONTRERAS D. ${ }^{2}$, JAIME CARTES U. ${ }^{3}$, \\ MÓNICA MARTÍNEZ M. ${ }^{3}$, CARLOS MARTÍNEZ P. ${ }^{4}$, VALERIA ALVARADO S. ${ }^{4}$, \\ MIRENTXU IRURETAGOYENA B. ${ }^{5}$, JORGE MARTÍNEZ C. ${ }^{6}$ \\ 1 Residente de Cirugía Plástica y Reconstructiva. \\ 2 Sección de Cirugía Plástica y Reconstructiva. \\ 3 Residente de Cirugía General. \\ 4 Laboratorio de Cirugía Experimental, Centro de Investigaciones Médicas, División de Cirugía. \\ Departamento de Reumatología. \\ 6 Programa de Trasplantes, Departamento de Cirugía Digestiva. \\ Facultad de Medicina, Pontificia Universidad Católica de Chile. \\ Santiago, Chile.
}

\section{Abstract}

\section{Basic microsurgical training for a vascularized composite allotransplantation animal model}

Introduction: Rat hind limb transplantation is a complex animal model of vascularized composite allotransplantation (VCA). A basic microsurgical training is required prior to the implementation of this model. Aim: To propose a training program for the acquisition of basic skills to perform a microsurgical VCA model. Animals and Methods: The training program was conducted in two stages. First, at the dry lab, basic suturing skills with 9-0 to 11-0 nylon sutures were practiced, reproduced from surgical videos performed by experts. In a second stage, at the wet lab using 13 Lewis rats, 6 hind limb microsurgical dissections were performed and the important steps for transplantation were identified: 10 end to end femoral artery anastomoses with 10-0 nylon interrupted suture; 10 end to end femoral vein anastomoses with 10-0 nylon suture; 3 femoral vein interposition in the femoral artery; 6 end to end sciatic nerve neurorrhaphy; 4 femur osteosynthesis with $21 \mathrm{~g}$ needle and wire cerclage. Anastomotic patency rate and anastomotic surgical time were recorded. Results: Arterial and venous patency rate was 100 and $90 \%$ respectively. Surgical time decreased from 49 to 24 minutes on arterial anastomoses and from 55 to 25 minutes on venous anastomoses after completion

*Recibido el 14 de octubre de 2012 y aceptado para publicación el 15 de abril de 2013.

Los autores no refieren conflictos de interés.

Fuente de financiamiento: Concurso Investigación para Residentes. División de Cirugía. Pontificia Universidad Católica de Chile.

Aprobado por el Comité de Ética Animal de la Dirección de Investigación de la Facultad de Medicina.

Correspondencia: Dr. Alejandro Ramírez M.

Marcoleta 350, Patio Interior, Edificio División de Cirugía. $2^{\circ}$ piso. Teléfono: 23543472.

aeramire@.puc.cl 
of the training program. When a vein interposition was performed, an immediate patency rate of $100 \%$ was obtained. Conclusion: A successful staged training model of basic microsurgical skills was performed, in order to perform a VCA model. models.

Key words: Vascularized composite allotransplantation, microsurgical training, rat hind limb, animal

\section{Resumen}

Introducción: El trasplante de extremidad posterior de la rata es un modelo microquirúrgico de alotrasplante compuesto vascularizado (ACV), que requiere para su implementación de un entrenamiento microquirúrgico básico sistematizado. Objetivo: Comunicar un modelo de entrenamiento microquirúrgico básico para adquirir las habilidades y destrezas que permitan realizar un modelo microquirúrgico de ACV. Animales y Métodos: El entrenamiento se realizó en 2 etapas: La primera, en laboratorio en seco. A partir de vídeos de microcirugía, se practicó las destrezas para maniobrar suturas de nylon de 9-0 a 11-0. En la segunda etapa in vivo con animales, (13 ratas Lewis), se realizaron: 6 disecciones microquirúrgicas de extremidad posterior para reconocimiento de estructuras anatómicas; 10 anastomosis término-terminal (T-T) de arteria femoral con sutura interrumpida 10-0; 10 anastomosis T-T de vena femoral; 3 interposiciones de vena femoral en arteria femoral; 6 neurorrafias T-T de nervio ciático; 4 fijaciones óseas de fémur con aguja $21 \mathrm{~g}$ y cerclaje con alambre. Se evaluó la permeabilidad de las anastomosis y los tiempos quirúrgicos. Resultados: Anastomosis arteriales: la permeabilidad inmediata fue de un $100 \%$. Los tiempos de anastomosis disminuyeron de 49 a 24 min con el entrenamiento. Anastomosis venosas: la permeabilidad inmediata fue de un 90\%. Los tiempos de anastomosis disminuyeron paulatinamente de 55 a $25 \mathrm{~min}$. Interposiciones venosas: se logró una permeabilidad de $100 \%$. Conclusión: Una estrategia de entrenamiento por etapas, es una forma factible de entrenamiento en microcirugía. Este entrenamiento permite obtener una aceptable permeabilidad anastomótica, paso crítico para realizar modelos de ACV.

Palabras clave: Tejido compuesto, trasplante, modelo animal, alotrasplante, extremidad posterior de la rata, microcirugía, entrenamiento microquirúrgico.

\section{Introducción}

Los alotrasplantes compuestos vascularizados (ACV) son la próxima frontera del campo de los trasplantes de órganos y tejidos ${ }^{1}$. En la última década los trasplantes de manos y de cara, constituyen las indicaciones más conocidas de esta nueva área de la medicina y de la cirugía ${ }^{2,3}$. Para su implementación clínica, el entrenamiento microquirúrgico en modelos animales es una etapa esencial a realizar. Las curvas de aprendizaje de las técnicas microquirúrgicas demandan habilidades básicas y de un entrenamiento continuo ${ }^{4}$. Sin embargo, frecuentemente los recursos para el entrenamiento son escasos y la seguridad de los pacientes limitan las oportunidades de docencia sistemática. Por otro lado, el entrenamiento en un laboratorio en seco sin animales, es cada vez más usado para desarrollar las habilidades y destrezas pertinentes en microcirugía, a la vez que disminuye los costos y la utilización de un gran número de animales de laboratorio ${ }^{5-19}$.

El trasplante de extremidad posterior de la rata, es un modelo animal microquirúrgico complejo de $\mathrm{ACV}^{20,21}$ que los autores pretenden implementar. Previo a la ejecución del mismo, se diseñó realizar un modelo de entrenamiento microquirúrgico bási- co combinando una práctica en laboratorio en seco con la ejecución de microanastomosis vasculares, neurales y osteosíntesis en extremidades posteriores en ratas. El objetivo del trabajo es comunicar un modelo por etapas de entrenamiento microquirúrgico y sus resultados en términos de permeabilidad de las microanastomosis vasculares realizadas y de los tiempos quirúrgicos de las mismas.

\section{Materiales y Métodos}

Se diseñó un programa de entrenamiento microquirúrgico en 2 etapas. Éste lo realizó uno de los autores, durante su residencia de Cirugía General, sin previa experiencia microquirúrgica, bajo la supervisión de un microcirujano experimentado. El protocolo de implementación fue aprobado por la Secretaría de Investigación de la División de Cirugía de la Facultad de Medicina de nuestra Universidad y la etapa de entrenamiento con animales, fue aprobada por el Comité de Ética Animal de la Dirección de Investigación de la misma Facultad.

\section{Entrenamiento microquirúrgico}

a) Primera etapa de laboratorio en seco: Se utilizó un microscopio binocular $\left(\right.$ Leyca $\left.^{\circledR}\right)$ con poder 
de aumento hasta $5 \mathrm{X}$ y suturas de nylon 9-0, 10-0, y 11-0. Se empleó además un teflón número 21 , un guante de látex y un molde se silicona. Como instrumentos microquirúrgicos, se usó una pinza de relojero número 5 , un microporta, una microtijera de disección y un microclamp aproximador (Figura1). Para el estudio de vídeos de microcirugía, se utilizaron vídeos del protocolo de estudio del Dr. Acland.

El entrenamiento se comenzó con el estudio de los vídeos de microcirugía, seguido por la práctica de destrezas microquirúrgicas básicas. Se practicaron 300 puntos simples más anudados con nylon 110. Durante la ejecución de este ejercicio, se practicó además la toma de la aguja, su posicionamiento en el porta y la recogida desde la silicona; 200 puntos simples más anudados con nylon 10-0; 20 suturas corridas con nylon 10-0 y 20 ligaduras simples con nylon 9-0 (Figura 2).

b) Segunda etapa, entrenamiento in vivo: Se utilizaron 13 ratas machos Lewis que pesaron entre 200 y $400 \mathrm{~g}$ que fueron anestesiadas con una mezcla de ketamina $(70 \mathrm{mg} / \mathrm{kg})+$ xilazina $(3 \mathrm{mg} / \mathrm{kg})$ intraperitoneal, además de los materiales utilizados en la etapa previamente descrita.

\section{Disección microquirúrgica}

El entrenamiento in vivo se comenzó con las disecciones microquirúrgicas de extremidad posterior (3 ratas, 6 disecciones) (Figura 3): durante esta etapa se adquirieron destrezas básicas de disección y se reconocieron las estructuras anatómicas fundamentales para realizar el trasplante: disección de vasos femorales, disección de nervio ciático, reconocimiento de los grupos musculares y del fémur.

\section{Anastomosis microvasculares}

En 10 ratas Lewis se practicó: (i) 10 anastomosis término-terminal de arteria femoral con sutura interrumpida de nylon 10-0. Cinco de éstas se realizaron con la técnica de pared anterior y pared posterior (Figura 4A) y cinco con la técnica de triangulación (Figura 4B). De forma resumida, la primera técnica consiste en colocar los dos primeros puntos separados en 180 grados, para luego completar la pared anterior con 3 puntos más, luego se da vuelta el microclamp aproximador con la anastomosis para colocar 3 puntos en la pared posterior, completando la anastomosis con 8 puntos en total. En la técnica de triangulación, los primeros 2 puntos se separan en 120 grados para luego colocar 2 puntos entre estos, con lo que se completa uno de los lados del triángulo. A continuación se da vuelta la anastomosis y se coloca un punto en el medio de la pared posterior. Este punto se tracciona para un lado, colocándose 2 puntos en la cara expuesta, luego se tracciona hacia el lado contrario y se colocan 2 puntos más, hasta

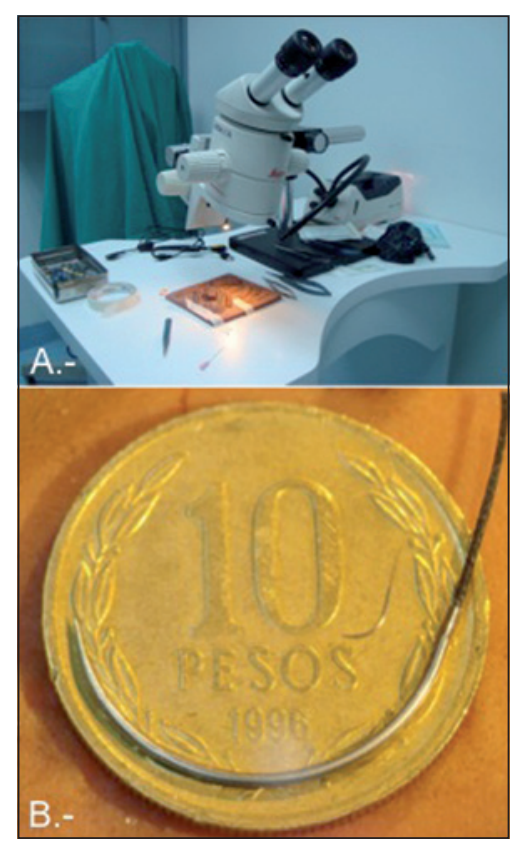

Figura 1. A) Material laboratorio en seco. B) Comparación entre una aguja 3-0 y una aguja microvascular 10-0.
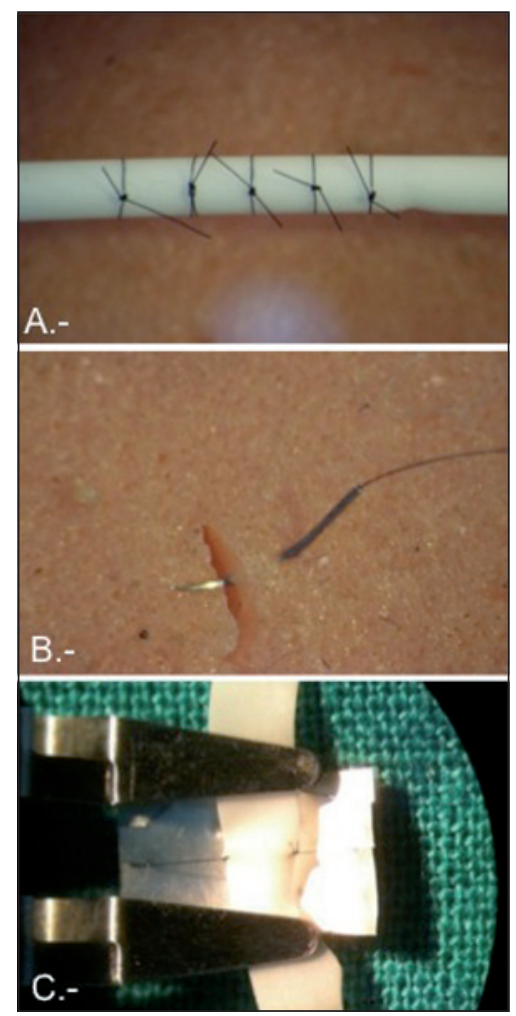

Figura 2. Entrenamiento microquirúrgico básico en laboratorio en seco. A) Anudado simple con nylon 9-0. B) Puntos simples en silicona. C) Montaje con microclamp aproximador, simulación con trozos de látex. 
completar la anastomosis con 9 puntos en total; (ii) Además se efectuó 10 anastomosis términoterminal de vena femoral con sutura interrumpida de nylon 10-0, con técnica de triangulación (Figuras 5 y 6 ), de la forma ya descrita para la arteria ${ }^{22}$. También se realizaron 3 interposiciones de vena femoral en la arteria femoral (Figura 7) (6 anastomosis término-

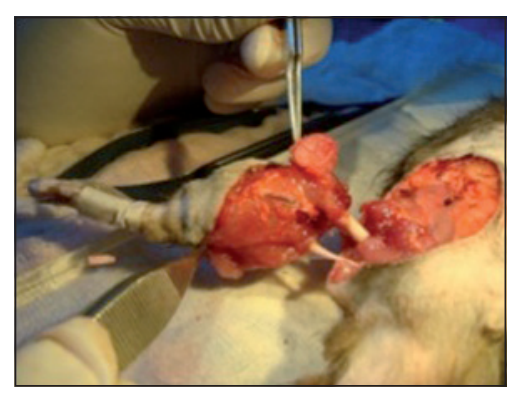

Figura 3. Disección extremidad posterior de rata Brown Norway. Se observan los vasos femorales seccionados (a), los músculos del muslo seccionados (b), el fémur (c) y el nervio ciático (d).

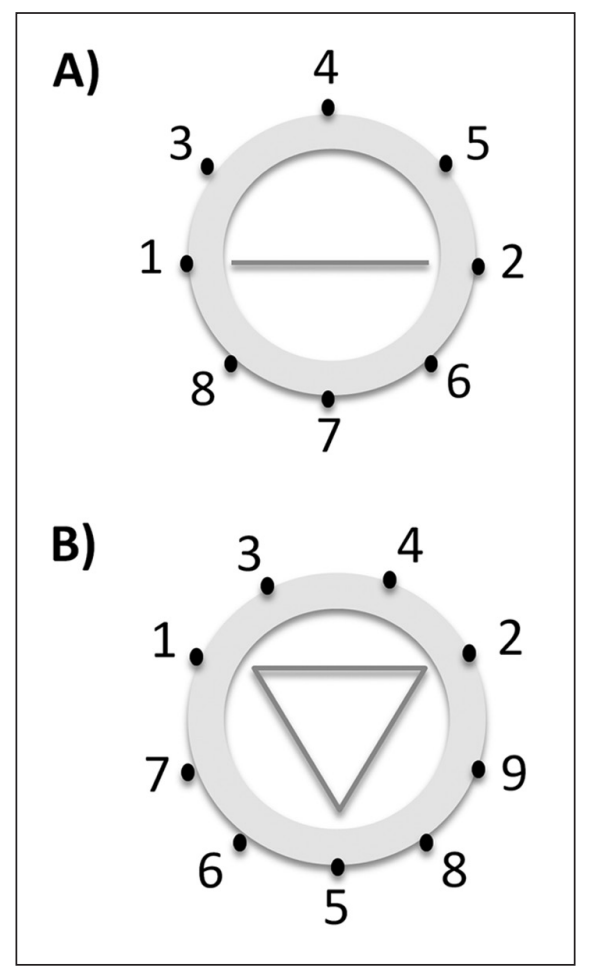

Figura 4. Esquema sobre las técnicas de anastomosis microvascular. La zona gris representa la pared del vaso sanguíneo. Los números muestran la ubicación y el orden de los puntos. A) Técnica de pared anterior y pared posterior. B) Técnica de triangulación. terminal arterio-venosas). Todas estas anastomosis, se realizaron bajo irrigación con solución de heparina no fraccionada $(100 \mathrm{UI} / \mathrm{ml})$. De los elementos no vasculares; (iv) se efectuaron 6 neurorrafias términoterminal de nervio ciático con sutura interrumpida de nylon 10-0 y (v) 4 osteosíntesis de fémur con aguja $21 \mathrm{~g}$, a modo de clavo endomedular más cerclaje con alambre de acero de $0,4 \mathrm{~mm}$ de diámetro.

En todas las anastomosis vasculares se evaluó la permeabilidad inmediata a los 2 y 30 min mediante inspección directa y test de Acland. El tiempo de anastomosis se cuantificó en todas las uniones arteriales y venosas.

\section{Resultados}

Fue factible realizar un entrenamiento por etapas. En total se realizaron 26 anastomosis microvasculares, siendo la permeabilidad anastomótica inmediata de $96,2 \%$. La permeabilidad inmediata de las anastomosis arteriales fue de un $100 \%$. La Tabla 1 muestra los tiempos de anastomosis microvascular

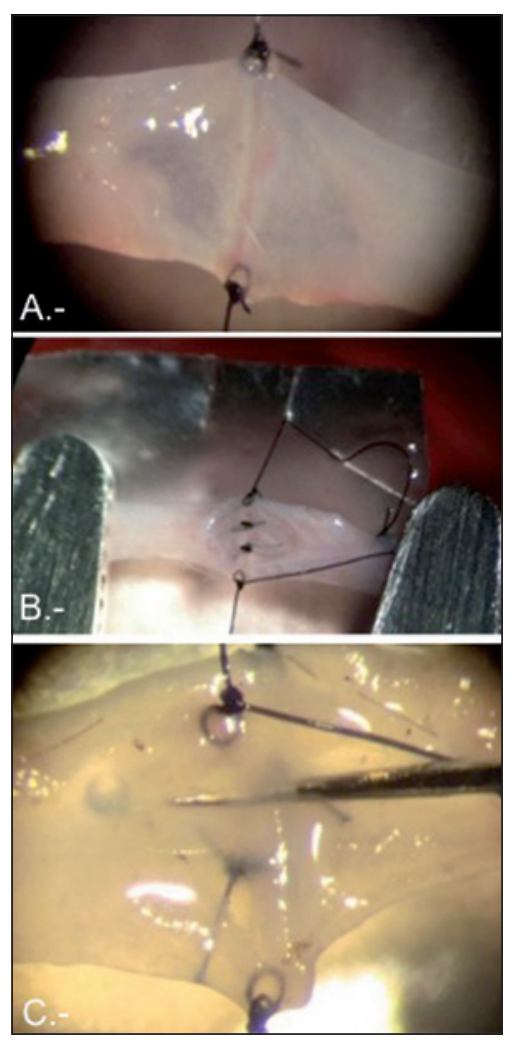

Figura 5. Práctica de anastomosis microvascular. Anastomosis de vena femoral con sutura interrumpida de nylon 10-0. Técnica de triangulación. 


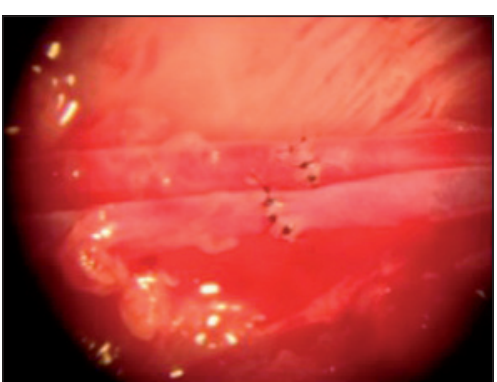

Figura 6. Anastomosis microvascular de arteria y vena femoral.

Tabla 1. Tiempos de anastomosis microvascular término-terminal de vasos femorales con nylon 10-0

\begin{tabular}{|ccc|}
\hline $\mathbf{n}$ & Anastomosis venosa & A. Arterial \\
1 & 55 & 49 \\
2 & 45 & 48 \\
3 & 43 & 44 \\
4 & 46 & 40 \\
5 & 44 & 32 \\
\hline 6 & 40 & 31 \\
7 & 30 & 36 \\
8 & 30 & 35 \\
\hline 9 & 29 & 30 \\
10 & 25 & 24 \\
\hline
\end{tabular}

n: número de anastomosis. Tiempo expresado en minutos

de las anastomosis venosas y arteriales. El tiempo de ejecución de la anastomosis arterial disminuyó paulatinamente de 49 a $24 \mathrm{~min}$. La permeabilidad inmediata de las anastomosis venosas fue de un $90 \%$. Observamos una trombosis en la primera anastomosis venosa en la evaluación a los $30 \mathrm{~min}$ sin identificarse un factor mecánico evidente (sutura de pared posterior, puntos desiguales, exposición de la media, etc.). El tiempo de anastomosis venosa disminuyó de 55 min en el primer caso a $25 \mathrm{~min}$ en el último. La permeabilidad anastomótica de las interposiciones venosas fue de un $100 \%$.

\section{Discusión}

El ACV consiste en el trasplante de diferentes tejidos complejos que pueden contener piel, músculo, hueso, cartílago, nervios, vasos sanguíneos, células inmunes o una combinación de estos ${ }^{1-3,23-25}$. Los modelos animales de ACV, permiten no sólo

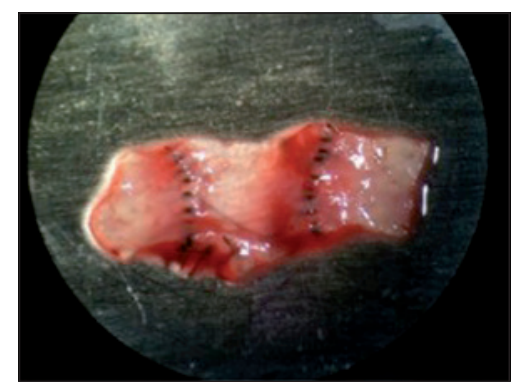

Figura 7. Interposición de vena femoral. Vaso abierto en forma longitudinal. Vista desde el lumen.

obtener entrenamiento microquirúrgico y estudiar la factibilidad técnica de estos trasplantes, sino también establecer plataformas para la investigación en $\mathrm{ACV}^{26}$. La dificultad técnica del ACV, requiere de cirujanos entrenados en diversas técnicas microquirúrgicas. En la literatura varios artículos describen los elementos necesarios para aprender microcirugía. Algunos de estos aspectos son la necesidad de tiempo, paciencia, motivación, tolerancia a la frustración y recursos entre otros $\mathrm{s}^{4,13,27-31}$. Si bien existen algunas publicaciones al respecto, aún no se ha desarrollado un currículo válido de entrenamiento para los especialistas que necesitan usar técnicas microquirúrgicas en su práctica diaria ${ }^{29,32-36}$. Entre los diferentes esquemas de entrenamiento propuestos, está la simple exposición directa a procedimientos microquirúrgicos, sin embargo, esta opción, además de ser riesgosa para los pacientes, se reserva sólo a centros con alto volumen microquirúrgico. Así, se ha sugerido que el entrenamiento microquirúrgico debe empezar en el laboratorio y una vez dominadas las habilidades básicas de microcirugía, dar el paso a la microcirugía clínica. Sin embargo, aún no se ha estandarizado el entrenamiento en el laboratorio, ni la forma de evaluarlo ${ }^{28,35,37,38}$. Si bien creemos que se debe trabajar en este punto, nos parece que la mejor opción es adaptarse a las condiciones locales para lograr adquirir las destrezas básicas de microcirugía.

Este artículo, describe cómo con elementos relativamente simples, se puede obtener un entrenamiento microquirúrgico básico fuera del pabellón. Este entrenamiento debe ser estandarizado para poder comparar resultados, la evolución de las habilidades adquiridas y facilitar la enseñanza microquirúrgica. Si bien en nuestro trabajo no se observaron los resultados a mediano plazo como se ha realizado en otros estudios ${ }^{37}$, en todas las anastomosis microvasculares, se evaluó la permeabilidad inmediata y a los $30 \mathrm{~min}$, lo que en general revela cualquier problema mayor en la ejecución de una anastomosis microvascular. 
El objetivo primario de nuestro modelo de entrenamiento fue adquirir las habilidades básicas para implementar un modelo experimental microquirúrgico de ACV, realizado por residentes de Cirugía General. Sin embargo, se revelan las evidentes ganancias secundarias de este modelo de entrenamiento como un primer paso, antes de realizar microcirugía en forma clínica.

Según la experiencia aquí presentada, creemos que un entrenamiento básico en el laboratorio, que incluya una etapa de laboratorio en seco, independiente del tipo o subetapas de entrenamiento, es una buena forma de iniciar el entrenamiento microquirúrgico para poder implementar en nuestro medio un modelo microquirúrgico complejo de ACV.

\section{Referencias}

1.- Swearingen B, Ravindra K, Xu H, Wu S, Breidenbach WC, Ildstad ST. Science of composite tissue allotransplantation. Transplantation 2008;86:627-35.

2.- Devauchelle B, Badet L, Lengele B, Morelon E, Testelin S, Michallet M, et al. First human face allograft: early report. Lancet 2006;368:203-9.

3.- Dubernard JM, Owen E, Herzberg G, Lanzetta M, Martin X, Kapila H, et al. Human hand allograft: report on first 6 months. Lancet 1999;353:1315-20.

4.- Cigna E, Bistoni G, Trignano E, Tortorelli G, Spalvieri C, Scuderi N. Microsurgical teaching: our experience. J Plast Reconstr Aesthet Surg. 2010;63:e529-31.

5.- Freys SM, Koob E. Education and training in microsurgery without experiments on live animals. Handchir Mikrochir Plast Chir. 1988;20:11-6.

6.- Steffens K, Koob E, Hong G. Training in basic microsurgical techniques without experiments involving animals. Arch Orthop Trauma Surg. 1992;111:198-203.

7.- Crosby NL, Clapson JB, Buncke HJ, Newlin L. Advanced non-animal microsurgical exercises. Microsurgery 1995; 16:655-8.

8.- Weber D, Moser N, Rosslein R. A synthetic model for microsurgical training: a surgical contribution to reduce the number of animal experiments. Eur J Pediatr Surg. 1997;7:204-6.

9.- Southern SJ, Ramakrishnan V. Dexter: a device for the assessment of microsurgical instrumentation and instruction of trainees. Microsurgery 1998;18:430-1.

10.- Yenidunya MO, Tsukagoshi T, Hosaka Y. Microsurgical training with beads. J Reconstr Microsurg. 1998;14:1978.

11.- Demirseren ME, Tosa Y, Hosaka Y. Microsurgical training with surgical gauze: the first step. J Reconstr Microsurg. 2003;19:385-6.

12.- Brackley PT, Nguyen DQ, Williams J, Roe G, Graham KE. A simple and effective adjunct to microsurgical training-the Whiston cup. J Plast Reconstr Aesthet Surg. 2006;59:1010-1.
13.- Furka I, Brath E, Nemeth N, Miko I. Learning microsurgical suturing and knotting techniques: comparative data. Microsurgery 2006;26:4-7.

14.- Inoue T, Tsutsumi K, Adachi S, Tanaka S, Saito K, Kunii N. Effectiveness of suturing training with 10-0 nylon under fixed and maximum magnification (x 20) using desk type microscope. Surg Neurol. 2006;66:183-7.

15.- Rayan B, Rayan GM. Microsurgery training card: a practical, economic tool for basic techniques. J Reconstr Microsurg. 2006;22:273-275; discussion 276.

16.- Dustagheer S, Brown AP. Synthetic latex conduits as an aid for microsurgical training. Plast Reconstr Surg. 2008; $122: 321$.

17.- Colebunders B, Matthew MK, Thomson JG. The use of a surgical glove in microsurgical training: a new point of view. Microsurgery 2010;30:505-6.

18.- Menovsky T. Microsurgical training model using chicken thigh. Plast Reconstr Surg. 2011;128:609-10.

19.- Sakrak T, Kose AA, Karabagli Y, Kocman AE, Ozbayoglu AC, Cetin C. Rat tail revascularization model for advanced microsurgery training and research. J Reconstr Microsurg. 2011;27:391-6.

20.- Shapiro RI, Cerra FB. A model for reimplantation and transplantation of a complex organ: the rat hind limb. J Surg Res. 1978;24:501-6.

21.- Doi K. Homotransplantation of limbs in rats. A preliminary report on an experimental study with nonspecific immunosuppressive drugs. Plast Reconstr Surg. 1979;64:613-21.

22.- Sumpter TL, Lunz Iii JG, Castellaneta A, Matta B, Tokita D, Turnquist HR, et al. Dendritic cell immunobiology in relation to liver transplant outcome. Front Biosci. (Elite Ed) 2009;1:99-114.

23.- Kann BR, Furnas DW, Hewitt CW. Past, present, and future research in the field of composite tissue allotransplantation. Microsurgery 2000;20:393-9.

24.- Tobin GR, Breidenbach WC, 3rd, Ildstad ST, Marvin MM, Buell JF, Ravindra KV. The history of human composite tissue allotransplantation. Transplant Proc. 2009;41:466-71.

25.- Wu S, Xu H, Ravindra K, Ildstad ST. Composite Tissue Allotransplantation: Past, Present and FutureThe History and Expanding Applications of CTA as a New Frontier in Transplantation. Transplant Proc. 2009;41:463-5.

26.- Siemionow M, Kulahci Y. Experimental models of composite tissue allograft transplants. Semin Plast Surg. 2007;21:205-12.

27.- Green CJ. Microsurgery in the clinic and laboratory. Lab Anim. 1987;21:1-10

28.- Zarabini AG, Galeano M. From surgical gloves to the rat. The various stages of microsurgery learning. Minerva Chir. 2000;55:687-92.

29.- Miko I, Brath E, Furka I. Basic teaching in microsurgery. Microsurgery 2001;21:121-3.

30.- Lahiri A, Lim AY, Qifen Z, Lim BH. Microsurgical 
skills training: a new concept for simulation of vesselwall suturing. Microsurgery 2005;25:21-4.

31- Martins PN, Montero EF. Basic microsurgery training: comments and proposal. Acta Cir Bras. 2007;22:79-81.

32.- Costoya A, Gomel V. The microsurgical training of the gynecologist. Rev Chil Obstet Ginecol. 1981;46:173-83.

33.- Korber KE. A basic microsurgical skills curriculum for the plastic surgeon. Plast Reconstr Surg. 1991;87:810-1.

34.- Scholz M, Mucke T, Holzle F, Schmieder K, Engelhardt M, Pechlivanis I, et al. A program of microsurgical training for young medical students: are younger students better? Microsurgery 2006;26:450-5.

35.- Chan WY, Matteucci P, Southern SJ. Validation of microsurgical models in microsurgery training and competence: a review. Microsurgery 2007;27:494-9.

36.- Lascar I, Totir D, Cinca A, Cortan S, Stefanescu A, Bratianu R, et al. Training program and learning curve in experimental microsurgery during the residency in plastic surgery. Microsurgery 2007;27:263-7.

37.- Ilie V, Ghetu N, Popescu S, Grosu O, Pieptu D. Assessment of the microsurgical skills: 30 minutes versus 2 weeks patency. Microsurgery 2007;27:451-4.

38.- Lin TS, Chiang YC. Correlation between microsurgical course performance and future surgical training selection by intern and junior residents. Microsurgery 2008;28:171-2. 